PoS $\quad \begin{aligned} & \text { PROCEEDINGS } \\ & \text { OF SCIENCE }\end{aligned}$

\title{
Unquenching the Landau Gauge Lattice Propagators and the Gribov Problem
}

\section{Paulo J. Silva* and Orlando Oliveira}

Centro de Física Computacional, Universidade de Coimbra, Portugal

E-mail: psilva@teor.fis.uc.pt, orlando@teor.fis.uc.pt

The gluon and ghost propagators are computed using both quenched and dynamical configurations for the same lattice spacings. The Wilson fermions are simulated at several quark masses. Furthermore, the effect of the Gribov copies is evaluated for all sets of configurations.

The XXVIII International Symposium on Lattice Field Theory

June 14-19,2010

Villasimius, Sardinia Italy

* Speaker. 


\section{Introduction and motivation}

It is well-known that the Faddeev-Popov (FP) procedure [1] can not be applied to the nonperturbative regime of Quantum Chromodynamics (QCD), due to the so-called Gribov copies problem. The FP trick assumes a unique solution of the gauge fixing condition on each set of gauge related configurations. Gribov [2] has shown, for the Landau and Coulomb gauges, that there are more than one configuration satisfying the gauge condition on each gauge orbit. Furthermore, it is not possible to define a local continuous gauge fixing condition free of Gribov copies [3, 4].

Many (quenched) lattice simulations have confirmed the existence of Gribov copies [5], and the influence of this issue on Landau gauge gluon and ghost propagators have been studied (see, for example, $[6,7])$. The simulations show that for the gluon propagator the influence of Gribov copies is null or small, whereas for the ghost propagator the effect is larger.

In this paper we recover the computation of gluon and ghost propagators on dynamical configurations, including an investigation of the Gribov copies. Similar studies have been performed by other groups while ago [8] but, to the best of our knowledge, no investigation on the influence of the Gribov copies in dynamical simulations is found in the literature.

\section{Lattice setup}

In this paper we will consider $16^{4}$ configurations. Three different sets of dynamical configurations for Wilson gauge action at $\beta=5.6$, using Wilson fermions, with different $\kappa$ 's, see table 1 , were considered. For the dynamical ensembles, the lattice spacing have been computed using the Sommer scale $r_{0}=0.5 \mathrm{fm}$. Data for $a r_{0}, m_{q}$ and $m_{\pi}$ have been taken from [9]. The $\beta$ values for the corresponding quenched ensembles have been computed using [10] so that they have the same lattice spacing as the dynamical counterparts. Quenched simulations have been performed using MILC code [11], whereas dynamical simulations have been performed using Chroma software library [12]; the MD integrator used in the HMC simulations has been tuned using the techniques described in [13].

Details on the lattice Landau gauge fixing and computation of the propagators can be found in [7]. The lattice data for the gluon propagator has been renormalized by requiring

$$
\left.D_{R}\left(q^{2}\right)\right|_{q^{2}=\mu^{2}}=\frac{1}{\mu^{2}}=Z_{R} D_{\text {Lat }}\left(\mu^{2}\right)
$$

at $\mu=3.5 \mathrm{GeV}$. In what concerns the ghost propagator, due to the very small statistical errors, we were unable to fit the high momenta in order to perform the renormalization. Given that we

\begin{tabular}{ccccccc}
\hline \multicolumn{3}{c}{ Quenched } & \multicolumn{4}{c}{ Dynamical Wilson } \\
$\beta$ & $\mathrm{a}(\mathrm{fm})$ & \# conf. & $\kappa$ & $\pi$ mass $(\mathrm{MeV})$ & $q$ mass $(\mathrm{MeV})$ & \# conf. \\
\hline 6.07 & 0.0828 & 60 & 0.1575 & 665 & 66 & 71 \\
6.0845 & 0.0809 & 66 & 0.1580 & 485 & 34 & 71 \\
6.106 & 0.0782 & 62 & 0.15825 & 385 & 22 & 71 \\
\hline
\end{tabular}

Table 1: Lattice setup. 




(a) Gluon propagator at $a=0.0828 \mathrm{fm}$.



(c) Gluon propagator at $a=0.0809 \mathrm{fm}$.

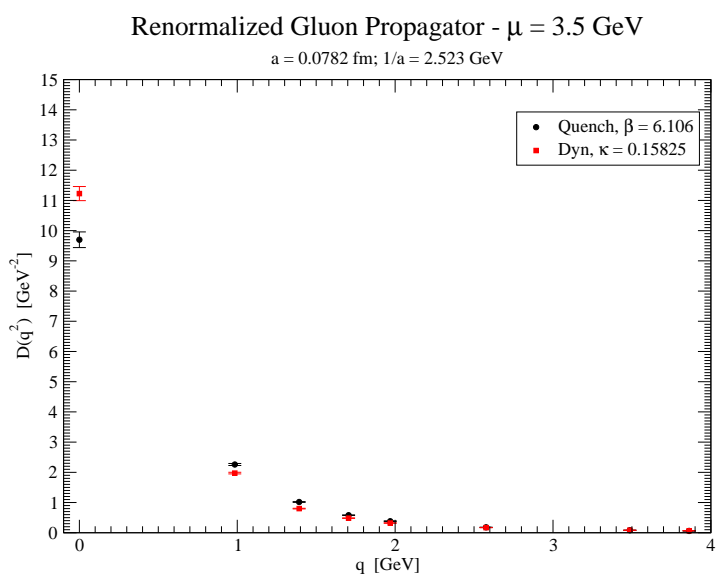

(e) Gluon propagator at $a=0.0782 \mathrm{fm}$.

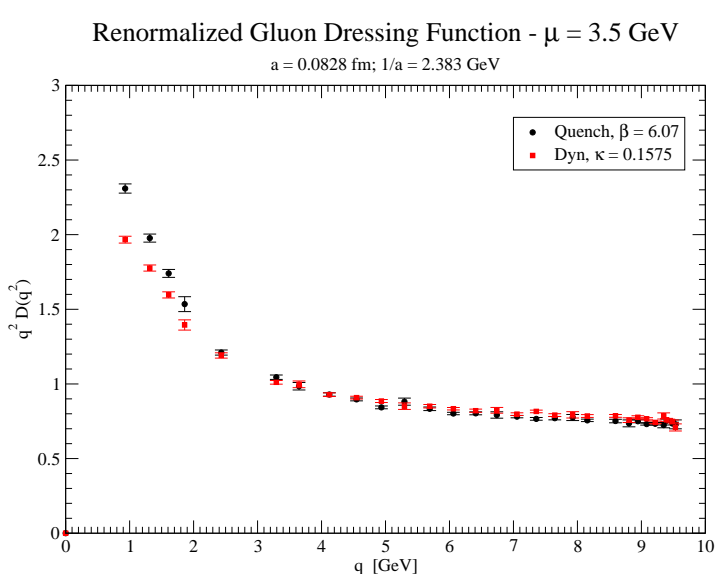

(b) Gluon dressing function at $a=0.0828 \mathrm{fm}$.

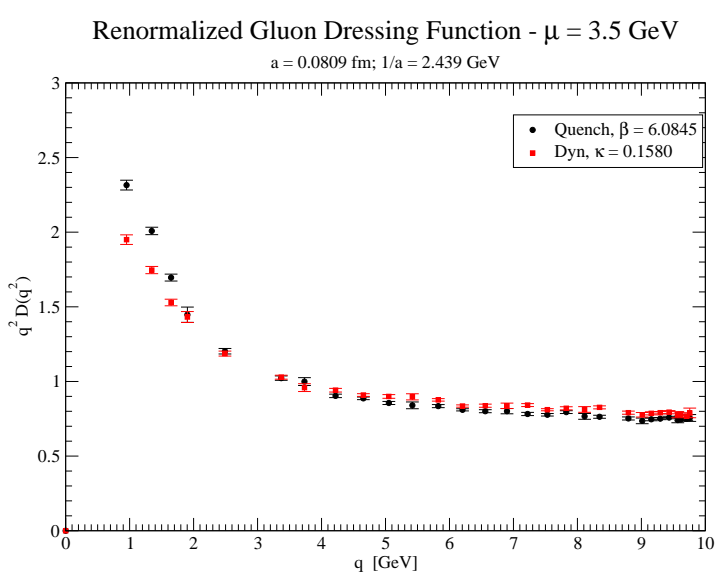

(d) Gluon dressing function at $a=0.0809 \mathrm{fm}$.

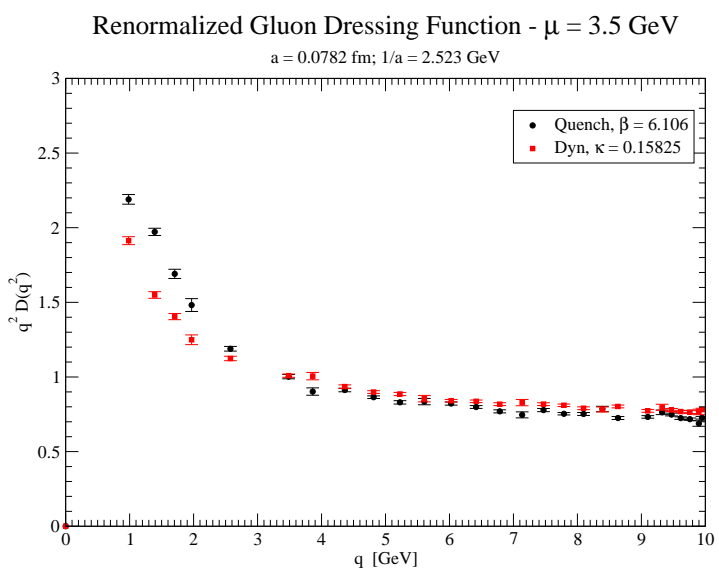

(f) Gluon dressing function at $a=0.0782 \mathrm{fm}$.

Figure 1: Dynamical gluon propagators and dressing functions versus quenched counterparts. 


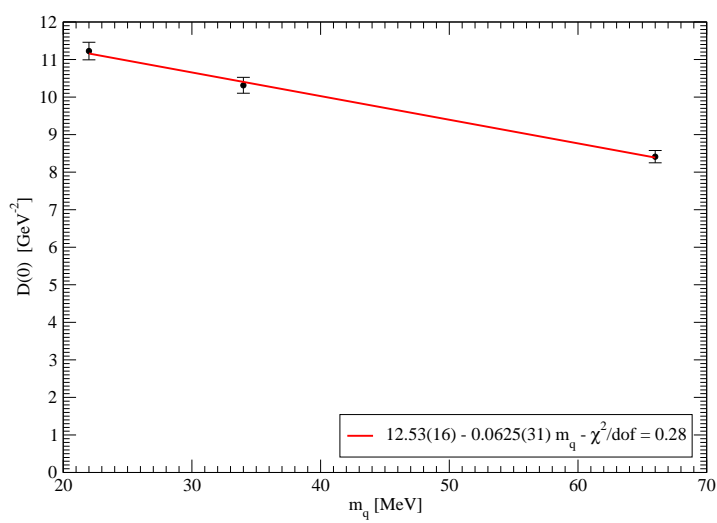

(a) $D(0)$ vs. $q$ mass

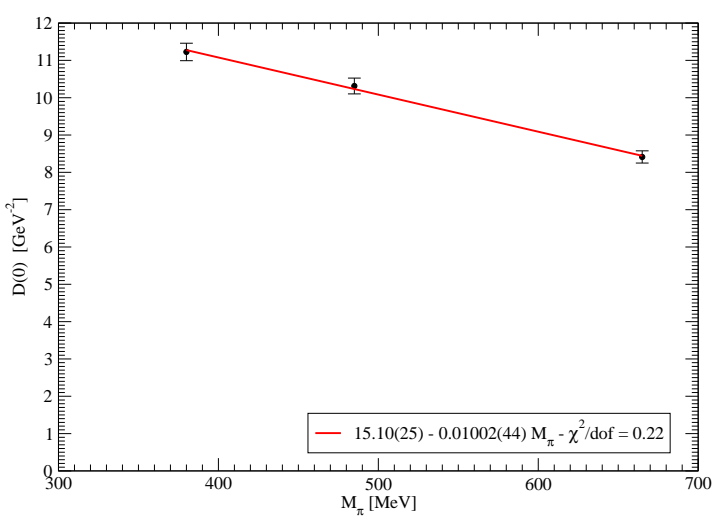

(b) $D(0)$ vs. $\pi$ mass.

Figure 2: Zero momentum gluon propagator as a function of quark and pion masses.

observed no difference in the UV between quenched and unquenched propagators, we just show the bare data.

In order to evaluate the effect of Gribov copies, we have generated 100 copies per gauge configuration. We will consider, besides a random copy, the copies which have the largest and smallest value of the gauge fixing functional.

\section{Results}

In figure 1 the gluon propagator computed with dynamical fermions is compared with the quenched propagator. Figure 1 shows clearly that the effect of dynamical quarks is mainly seen in the infrared region. With the exception of the zero momentum, the propagator for the dynamical configurations is suppressed in the infrared. On the other hand, $D(0)$ from the dynamical ensembles is suppressed for heavier quarks, and enhanced for lighter quarks. Furthermore, $D_{d y n}(0)$ follows a linear behaviour as a function of the quark and pion masses - see figure 2 .

In what concerns the ghost propagator, see figure 3, the effect of dynamical configurations is again more important at low momenta, with the dynamical quarks enhancing the propagator in the infrared region, if compared with quenched results. The data also shows that the enhancement goes with the quark mass, i.e. the larger the quark mass, the larger is the enhancement.

The influence of the Gribov copies in the propagators is resumed in figures 4 and 5. Indeed, the pattern observed for the quenched and unquenched theories is similar: no or small effects for the gluon propagator; larger effects for the ghost propagator. Note that the Gribov effects in the ghost propagator are seen over a large range of momenta, with the propagator being suppressed for larger F maxima.

\section{Conclusions}

We have presented a first study of the effect of Gribov copies in Landau gauge gluon and ghost propagators computed from dynamical configurations. The results show that the effect is 
very similar for quenched and dynamical propagators. Furthermore, we have revisited the effects of dynamical quarks in gluon and ghost propagators.

\section{Acknowledgments}

P. J. Silva acknowledges support from FCT via grant SFRH/BPD/40998/2007. O. Oliveira acknowledges support from FAPESP. The authors acknowlegde financial support from FCT under contracts PTDC/FIS/100968/2008 and CERN/FP/109327/2009.

\section{References}

[1] L. P. Faddeev, V. N. Popov, Phys. Lett. B25 (1967) 29.

[2] V. N. Gribov, Nucl. Phys. B139 (1978) 1.

[3] I. M. Singer, Comm. Math. Phys. 60 (1978) 7.

[4] T. P. Killingback, Phys. Lett. B138 (1983) 87.

[5] See, for example, E. Marinari, C. Parrinello, Nucl. Phys. B362 (1991) 487; P. Marenzoni, P. Rossi, Phys. Lett. B311 (1993) 219.

[6] A. Cucchieri, Nucl. Phys. B508 (1997) 353, arXiv: hep-lat/9705005; P. J. Silva, O. Oliveira, Nucl. Phys. B690 (2004) 177, arXiv: hep-lat / 0403026 ; A. Sternbeck, E.-M. Ilgenfritz, M. Müller-Preussker, A. Schiller, Phys. Rev. D72 (2005) 014507, arXiv: hep-lat / 0506007 ; I. L. Bogolubsky, G. Burgio, M. Müller-Preussker, V. K. Mitrjushkin, Phys. Rev. D74 (2006) 034503, arXiv: hep-lat / 051105 6; I. L. Bogolubsky, V. G. Bornyakov, G. Burgio, E. M. Ilgenfritz, M. Müller-Preussker, V. K. Mitrjushkin, Phys. Rev. D77 (2008) 014504, arXiv:0707.3611[hep-lat].

[7] P. J. Silva, O. Oliveira, PoS (LAtTICE 2007) 333, arXiv:0710.0669 [hep-lat].

[8] P. O. Bowman, U. M. Heller, D. B. Leinweber, M. B. Parappilly, A. G. Williams, Phys. Rev. D70 (2004) 034509; A. Sternbeck, arXiv: hep-lat / 0609016 ; S. Furui, H. Nakajima, Phys. Rev. D73 (2006) 094606; P. O. Bowman, U. M. Heller, D. B. Leinweber, M. B. Parappilly, A. Sternbeck, L. von Smekal, A. G. Williams, J. Zhang, Phys. Rev. D76 (2007) 094505; W. Kamleh, P. O. Bowman, D. B. Leinweber, A. G. Williams, J. Zhang, Phys. Rev. D76 (2007) 094501.

[9] C. Urbach, K. Jansen, A. Shindler, U. Wenger, Comp. Phys. Comm. 174 (2006) 87.

[10] S. Necco, R. Sommer, Nucl. Phys. B622 (2002) 328, arXiv: hep-lat/ 0108008.

[11] http://www.physics.indiana.edu/ sg/milc.html.

[12] R. G. Edwards and B. Joó (SciDAC Collaboration and LHPC Collaboration and UKQCD Collaboration), Nucl. Phys. Proc. Suppl. 140 (2005) 832.

[13] M. A. Clark, B. Joó, A. D. Kennedy, P. J. Silva, PoS (Lattice 2010) 323, arXiv: 1011.0230 [hep-lat]. 


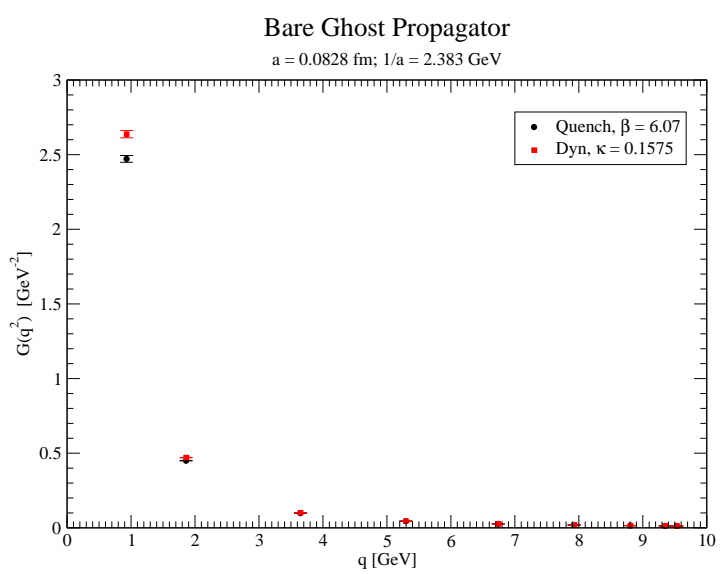

(a) Ghost propagator at $a=0.0828 \mathrm{fm}$.

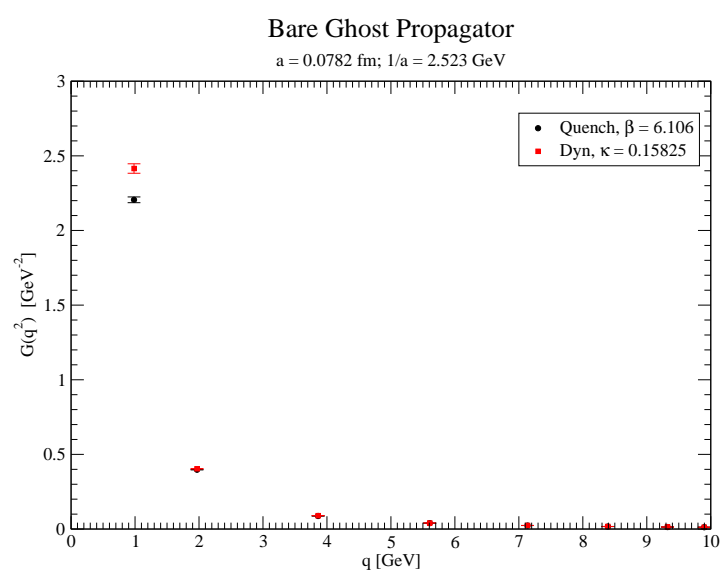

(c) Ghost propagator at $a=0.0782 \mathrm{fm}$.



(b) Ghost propagator at $a=0.0809 \mathrm{fm}$.

Figure 3: Dynamical bare ghost propagators versus quenched counterparts. 


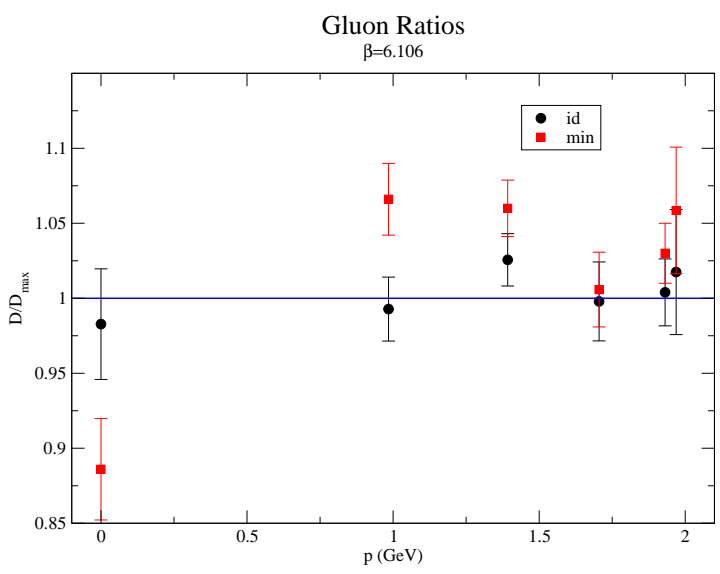

(a) Quenched gluon ratios at $\beta=6.106$.

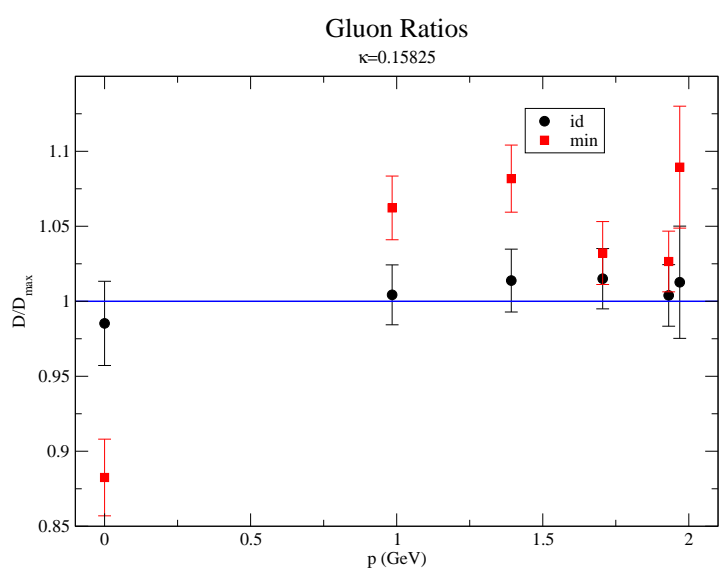

(b) Dynamical gluon ratios at $\kappa=0.15825$.

Figure 4: Gluon ratios.

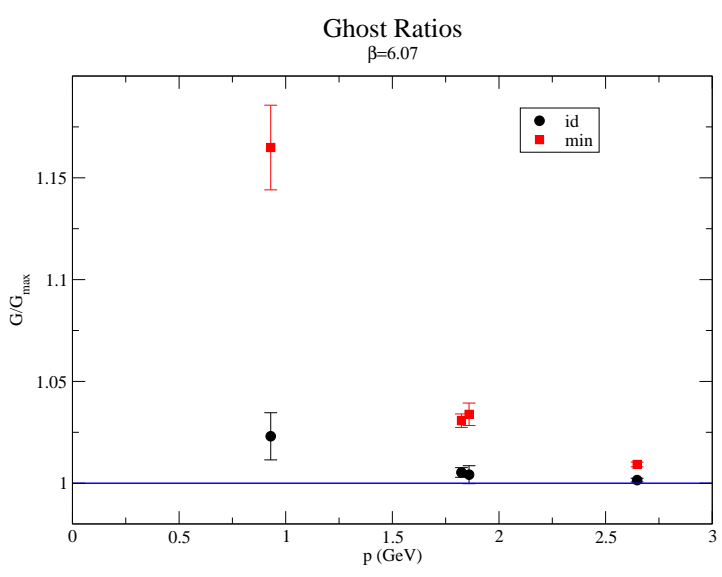

(a) Quenched ghost ratios at $\beta=6.07$.



(b) Dynamical ghost ratios at $\kappa=0.1575$.

Figure 5: Ghost ratios. 